\title{
Side-polished SMS based RI sensor employing macro-bending perfluorinated POF
}

\author{
Xuezhi Zhang 1,2,3, Boyue Yang1,2,3, Junfeng Jiang ${ }^{1,2,3 *}$, Kun Liu ${ }^{1,2,3}$, \\ Xiaojun Fan ${ }^{1,2,3}$, Zhaozhu Liu ${ }^{1,2,3}$, Min Peng ${ }^{1,2,3}$, Guanlong Chen ${ }^{1,2,3}$ and \\ Tiegen Liu ${ }^{1,2,3 *}$
}

A refractive index $(\mathrm{RI})$ sensor based on perfluorinated plastic optical fiber (PF-POF) is introduced in this paper. The PFPOF as multi-mode fiber was side-polished (SP) to form a macro-bending single-mode-multimode-single-mode (SMS) structure. Both ends of the sensor were closely connected to single-mode quartz optical fiber (SMF). The spectral characteristics of the sensor are measured, analyzed and discussed. The results show that when the length of PF-POF is 8 $\mathrm{cm}$, the macro-bending radius is $3 \mathrm{~cm}$, and the SP-depth is $20 \mu \mathrm{m}$. The intensity sensitivity reaches $-219.504 \mathrm{dBm} / \mathrm{RIU}$ in the range of $R I=1.330 \sim 1.356$. A reference is provided for the application of PF-POF in RI sensor in the future. The sensor is featured with low-cost, good flexibility and high efficiency.

Keywords: refractive index sensor; perfluoropolymer fiber; optical sensing; plastic optical fiber

Zhang XZ, Yang BY, Jiang JF, Liu K, Fan XJ et al. Side-polished SMS based RI sensor employing macro-bending perfluorinated POF. Opto-Electron Adv 4, 200041 (2021).

\section{Introduction}

Fluoropolymer is a straight chain alkane polymer that some or all hydrogen atoms of molecular structure are replaced by fluorine. Fluoropolymer is believed to be a preferred material for the high chemical resistance, barrier property, non-moisture absorption and weather resistance to prepare optical fiber cable, chemical resistant lining, electronic components, coating material and weather resistant film ${ }^{1}$. Compared with quartz optical fiber, PF-POF has many advantages, including good flexibility, low-cost and easy to operate. Compared with PMMAPOF, the lower water absorption of PF-POF makes it is more suitable for working in water or water solution ${ }^{2}$. The advantages of PF-POF are suitable for developing sensors with better performance. It has great potential in the field of developing optical fiber sensing, which is worthy of further research.

RI, an inherent optical property, can directly reflect concentration, purity, viscosity and other information of the solution. Therefore, it has been applied in various fields such as medical diagnosis and chemical analysis ${ }^{3,4}$. The mode interference fiber optic sensor has the advantages of anti-electromagnetic interference and real-time on-line measurement compared with the traditional RI sensing method, including critical angle method, Abbe refractometer and spectrometer. It is an effective means to measure the RI of the solution, and has been studied with growing interests in recent years. At present, there

${ }^{1}$ School of Precision Instrument and Opto-Electronics Engineering, Tianjin University, Tianjin 300072, China; ${ }^{2}$ Tianjin Optical Fiber Sensing Engineering Center, Institute of Optical Fiber Sensing of Tianjin University, Tianjin 300072, China; ${ }^{3}$ Key Laboratory of Opto-electronics Information Technology (Tianjin University), Ministry of Education, Tianjin 300072, China. "Correspondence: JF Jiang, E-mail: jiangjjjxu@tju.edu.cn; TG Liu, E-mail: tgliu@tju.edu.cn Received: 27 July 2020; Accepted: 2 October 2020; Published online: 25 October 2021 
are many types of fiber RI sensors reported, such as surface plasmon resonance $(\mathrm{SPR})^{5,6}$, corrosion $\mathrm{SMS}^{7,8}$, and fiber grating ${ }^{9-11}$. However, SPR needs to be coated, and the process is rather complicated; the depth of corrosion cannot be accurately controlled. In addition, hydrogen fluoride corrosion agent is toxic; the price of fiber grating is high, and the quartz fiber is fragile. As a result, extra care is required during the production of the sensor.

The maximum core RI of Graded-index PF-POF (Giga-POF50SR) of 1.356 and the cladding RI of 1.342. PF-POF can be well connected with quartz fiber for low loss, high bandwidth and flexibility. In addition, fluorine molecule has low volatility, which makes the fiber remain stable at high temperature ${ }^{12}$. In terms of practicability, compared with taper ${ }^{13,14}$ and etched SMS ${ }^{15}$, the sensor can work stably in water for a long time due to the development and utilization of new material perfluoropolymer, which is obviously practical in the field of liquid detection. In terms of performance, compared with thin core fiber ${ }^{16}$ and no-core fiber ${ }^{17}$, the sensor has high sensitivity. With the development of perfluoropolymers, the sensitivity of the sensor will be further improved, and the measurement range will also increase with the core refractive index.

In this paper, SMF and new material PF-POF are used to make SMS structure. On the basis of SMS structure, $\mathrm{PF}-\mathrm{POF}$ is polished by grindstone. Different from the Vgroove polishing technology ${ }^{18}$, grindstone polishing is a simple and effective method, which can flexibly control the SP-length and SP-depth. The main parameters that affect the sensitivity of the sensor are studied. The RI sensor with optimal parameters has the characteristics of high flexibility, simple processing, high sensitivity and low cost.

\section{Principle}

The light in the untreated PF-POF cannot penetrate the cladding and interact with the external environment. Therefore, it is necessary to improve the sensitivity of optical fiber by SP. In order to improve the performance of the sensor, the model is established as shown in Fig. 1(a) to study the influence of fiber structure modification on the sensitivity. From left to right, SP-area can be divided into two parts: the area where the core diameter gradually decreases and increases. Because the SP-area is symmetric, only the light guiding properties of the area with decreased diameter are analyzed. The radius of the clamping wheel is about $15 \mathrm{~cm}$, which is much larger than that of PF-POF. Therefore, the SP-radian drop and rise can be equivalent to drop or rise linearly, as shown in the red dotted line in the Fig. 1(a). The measurement principle of the sensor and the key parameters affecting sensitivity are analyzed from following two aspects.
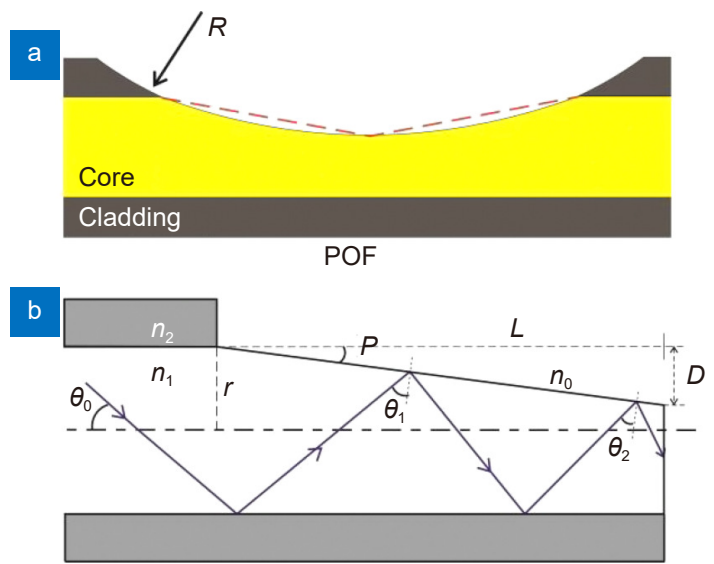

Fig. 1 | Schematic diagram of SP-area. (a) Structural equivalence. (b) Optical transmission.

\section{Evanescent-wave}

When total internal reflection occurs in the fiber, the light wave is not absolutely reflected back to the core, but penetrates into the surface of the cladding layer (about one wavelength), propagates along the interface for a short distance, and finally returns to the core. The wave passing through the cladding interface is called evanescent wave ${ }^{19}$. The evanescent depth is expressed as follows:

$$
d_{0}=\frac{\lambda}{2 \pi} \frac{1}{\sqrt{n_{1}^{2} \sin ^{2} \theta-n_{2}^{2}}},
$$

where $\lambda$ is the wavelength, $n_{1}$ is the core RI, and $n_{2}$ is the cladding RI. The incident angle at the core-cladding interface is called $\theta$ and the SP-depth is $D$. In this SP-mod$\mathrm{el}$, the cladding is removed and the external solution acts as a new cladding. Figure 1(b) describes the light propagation path in the SP-area. $\alpha$ is the slope angle of the SP-surface, $\theta_{0}$ is the angle between the light and the core center line, and $L$ is the SP-length of the falling area. The incident angle of light at the SP-interface for the first time (hereinafter referred to as the incident angle) is $\theta_{1}$, and the incident angle for the $i^{\text {th }}$ time is recorded as $\theta_{i}$. Then, $\theta_{i}$ can be expressed as:

$$
\theta_{i}=\frac{\pi}{2}-\theta_{0}-i P
$$

It can be seen from Eq. (1) that when $n_{1}>n_{2}$, the evanescent depth is increasing with the increased external environment RI, and the spectral intensity decreases 
continuously with the increase of the penetration depth. This means that the change of external solution RI can be detected from the change of transmission spectrum intensity.

With the decrease of the incident angle and the increase of the SP-length, the evanescent wave effect is enhanced. According to Eq. (2), the incident angle is determined by SP-depth and SP-radian. Therefore, the sensitivity of the sensor can be improved by properly increasing the SP-depth, SP-radian and SP-length. It should be noted that the enhancement of evanescent wave effect caused by the increase of SP-length, SP-depth and SP-radian will lead to the decrease of the overall intensity of the output spectrum.

\section{Refraction effect}

In the SP-model, the external environment becomes a new cladding, and the transmitted light will refract under certain conditions. According to Fresnel equation and electromagnetic theory ${ }^{20}$, it can be concluded that the reflected light intensity $I^{\prime}$ and the refracted light intensity $I^{\prime \prime}$ are:

$$
\begin{gathered}
I^{\prime}=\left(\frac{\cos \theta-\sqrt{n_{01}^{2}-\sin ^{2} \theta}}{\cos \theta+\sqrt{n_{01}^{2}-\sin ^{2} \theta}}\right)^{2} I_{\mathrm{S}} \\
+\left(\frac{n_{01}^{2} \cos \theta-\sqrt{n_{01}^{2}-\sin ^{2} \theta}}{n_{01}^{2} \cos \theta+\sqrt{n_{01}^{2}-\sin ^{2} \theta}}\right)^{2} I_{\mathrm{P}}, \\
I^{\prime \prime}=\frac{\sqrt{n_{01}^{2}-\sin ^{2} \theta}}{\cos \theta}\left(\frac{2 \cos \theta}{\cos \theta+\sqrt{n_{01}^{2}-\sin ^{2} \theta}}\right)^{2} I_{\mathrm{S}} \\
+\frac{\sqrt{n_{01}^{2}-\sin ^{2} \theta}}{\cos \theta}\left(\frac{\cos \theta}{\sqrt{n_{01}^{2}-\sin ^{2} \theta}+n_{01}^{2} \cos \theta}\right)^{2} I_{\mathrm{P}},
\end{gathered}
$$

where $n_{01}$ is the relative RI of the external environment and the core, and $\theta$ is the incident angle. The incident light is decomposed into light with S-polarization and Ppolarization whose electric vector is perpendicular to and parallel to the incident plane. The light intensity of them is expressed by $I_{\mathrm{S}}$ and $I_{\mathrm{P}}$, respectively. If the intensity of incident light is $1 \mathrm{~mW}, I_{\mathrm{S}}=I_{\mathrm{P}}=0.5 \mathrm{~mW}$. The relationship between the intensity of reflected light and refracted light and the relative RI is shown in Fig. 2.

It can be seen from Fig. 2 that when $\sin \theta \geq n_{01}$, total internal reflection occurs, and the reflected light intensity is $1 \mathrm{~mW}$. When $\sin \theta<n_{01} \leq 1$, with the increase of $\mathrm{n}_{01}$, the intensity of reflected light begins to decrease; when $n_{01}=1$, the intensity becomes zero. In this process, the change rate gradually decreases; when $n_{01}>1$, the reflected light intensity increases from zero, and the slope

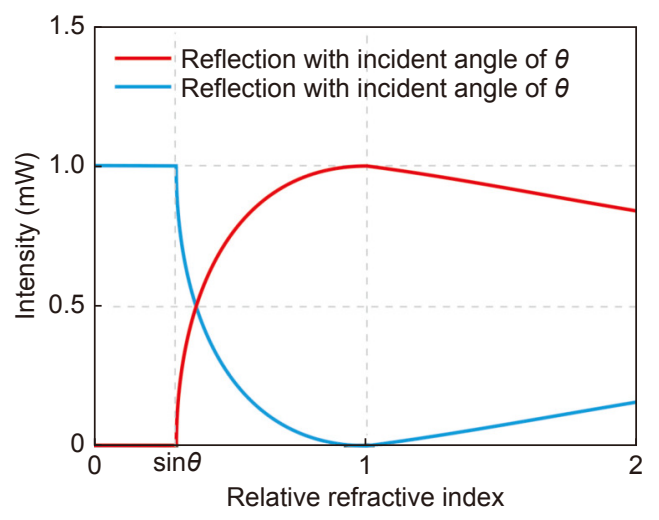

Fig. 2 | The relationship between the energy of reflected and refracted light and the relative $R I$ when the incident angle is $\theta$.

of increasing is small. Therefore, the change of external environment RI can be detected from the intensity change of transmission spectrum. Different incident angle will induce different reflected light intensity, but no matter what the incident angle is $\left(\theta<90^{\circ}\right)$, when the relative RI decreases from 1 , the reflected light intensity always increases with the decrease of relative RI.

The relationship between reflected light intensity and incident angle is shown in Fig. 3. Either $n_{1}<n_{0}$ or $n_{1}>$ $n_{0}$, with the increase of incident angle, the reflected light intensity will increase, and the refracted light intensity will decrease. In addition, the refraction effect area increases with the increase of the SP-length $L$. Therefore, the sensor sensitivity can be improved by increasing the SP-depth, SP-radian and SP-length.

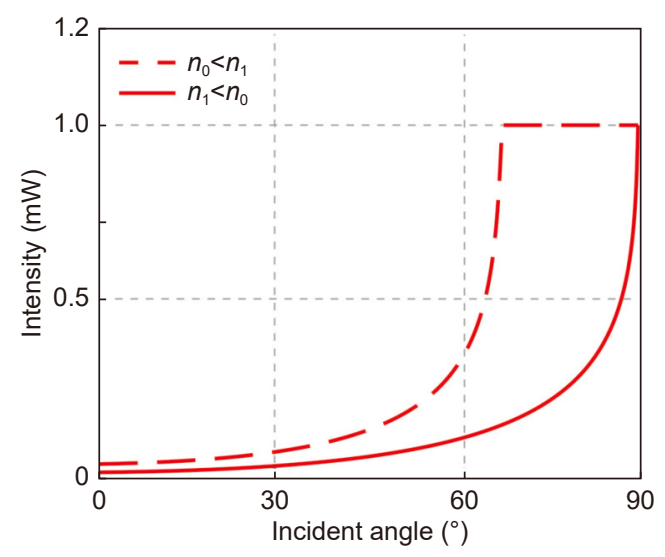

Fig. 3 | The variation of reflected light intensity with incident angle; the dotted line indicates $n_{01}=0.9$, and the solid line indicates $n_{01}=1.1$.

\section{Experimental and preparation}

As shown in Fig. 4, the experimental setup contains ASE broadband light source, spectrometer, RI sensor and glycerin aqueous solution with different RI. The cross section 
of PF-POF is shown in Fig. 5(a). Regarding connecting PF-POF to SMF or instruments effectively and reliably, the traditional method, UV curing bonding, is reliable. But this method is time-consuming, and highly dependent on the operator experience. Here, the butt joint method of ceramic sleeve ${ }^{21}$ is applied, and the connection diagram is shown in Fig. 5(b). This method ensures the accurate connection of optical fiber with low connection loss. Besides, this method is easy to operate and time-saving. Finally, the joint gap of the sleeve is coated with epoxy resin $\mathrm{AB}$ glue to avoid solution penetration.

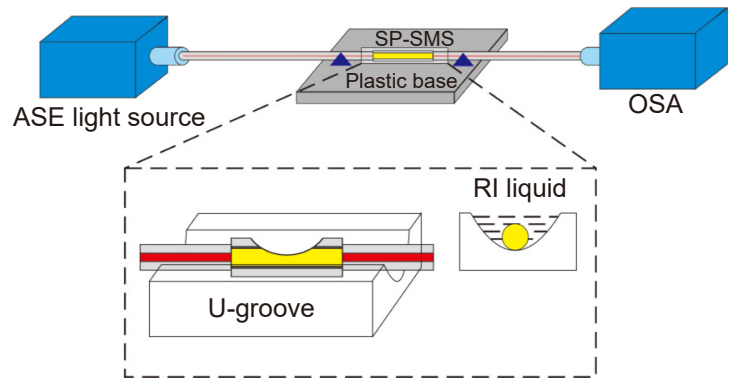

Fig. 4 | Schematic diagram of the experimental setup.
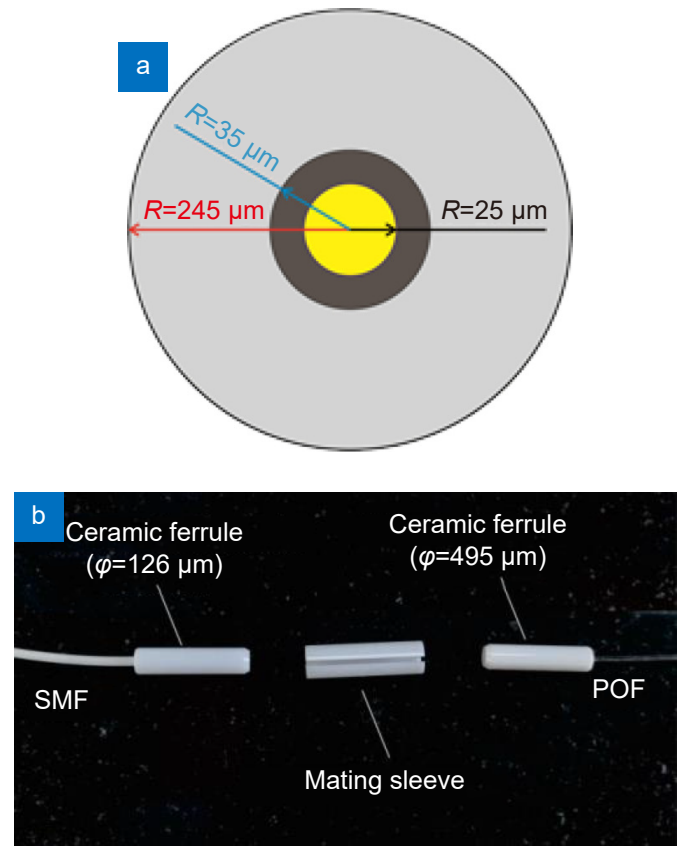

Fig. 5 I (a) Cross section of the POF. (b) Connection between POF and SMF using ceramic ferrule and ceramic mating sleeve.

At present, the main methods to make optical fiber sensitive to external RI are as follows: $\operatorname{taper}^{13,14}$, no-core fiber $^{22}$, D-shaped ${ }^{23}$, Microbend ${ }^{24}$ and hydrogen fluoride corrosion $^{18}$. However, all of the above methods have some defects. The taper of optical fiber is difficulted to control and the optical fiber gets thinned and is fragile after being tapered. For no-core fiber, in order to im- prove the sensitivity of sensor, a thin film is needed to be coated on the surface of the fiber. D-shaped and microbend are complex in operation. The corrosion depth is difficult to control for hydrogen fluoride corrosion.

The precision polishing device is shown in Fig. 6, PFPOF is fixed on the semicircular clamping wheel. The ends of POF is connected by optical power meter and light source. Whether the fiber core is polished is determined by optical power measured by a power meter when the move of clampling wheel is controlled by the guide. At this time, the descending distance at the other end of the guide is recorded, which is the SP-depth by microscope after polishing.

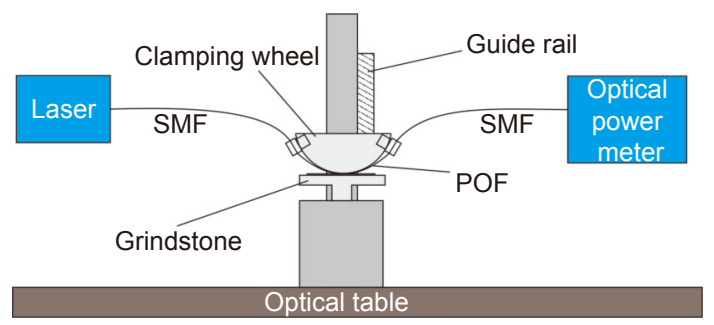

Fig. 6 | Schematic of experimental setup for polishing fibers.

\section{Experimental analysis}

The transmission spectrum of multimode interference in SMS is related to the length of multimode fiber ${ }^{21}$. SPtreatment can only slightly reduce the overall intensity of transmission spectrum, and will not affect the waveform. Whether the characteristic wavelength can be quickly found is one of the factors to evaluate the performance of sensor. The length of PF-POF can change the shape of spectrum by affecting the mode interference of light field, thus affecting the difficulty of peak finding and sensing performance, so the length characteristics of PFPOF are explored. The relationship between the length of PF-POF and transmission spectrum is studied. Fig. 7 shows the transmission spectra of PF-POF with length of $5 \mathrm{~cm}, 6 \mathrm{~cm}, 7 \mathrm{~cm}, 8 \mathrm{~cm}$ and $9 \mathrm{~cm}$ respectively. Due to the obvious multimode interference effect, complex interference spectrum appears. With the change of PF-POF length, the interference fringes also change. As shown in the red dotted box in Fig. 7, when the length of PF-POF is $8 \mathrm{~cm}$, the spectral notch depth is more obvious, which is suitable for the measurement of RI.

Since the radius of the side wheel is determined in this experiment, the SP-length is positively correlated with the SP-depth, so only the SP-depth is explored. The sensor is explored under the conditions of different SPdepth. Figure 8 shows the transmission spectra in air and 


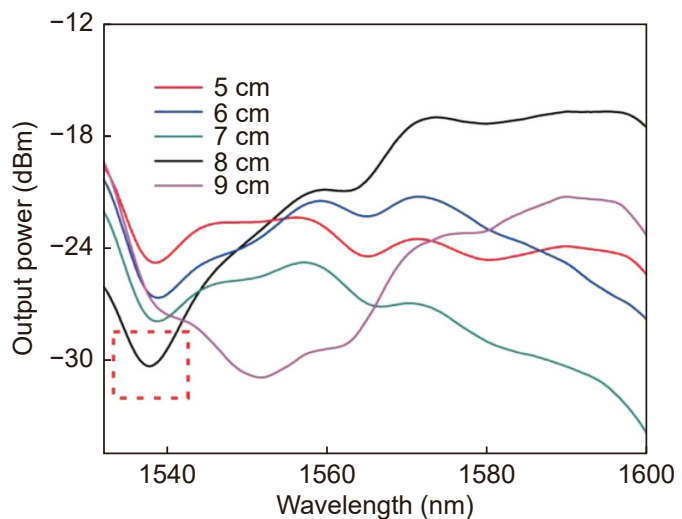

Fig. 7 | Transmission spectra of the RI sensor with different POF length.

glycerin aqueous solution $(R I=1.356)$ when the PF-POF length is $8 \mathrm{~cm}$ and the SP-depth $D$ is $0 \mu \mathrm{m}, 10 \mu \mathrm{m}, 20 \mu \mathrm{m}$ and $30 \mu \mathrm{m}$ respectively. The difference of RI between air and glycerin aqueous solution is 0.356 . In this situation, the characteristic wavelength intensity drift is $3.873 \mathrm{dBm}$ when $D=0 \mu \mathrm{m}, 5.267 \mathrm{dBm}$ when $D=10 \mu \mathrm{m}$, and 6.985 $\mathrm{dBm}$ when $D=20 \mu \mathrm{m}$. Therefore, it can be concluded that the sensor sensitivity can be improved by properly increasing the SP-depth. Figure 8(d) shows that the SPdepth is not good to be too large. When $D=30 \mu \mathrm{m}$, due to the large SP-depth, the fiber core is so thin that the optical energy loss is too high and the signal-to-noise ratio
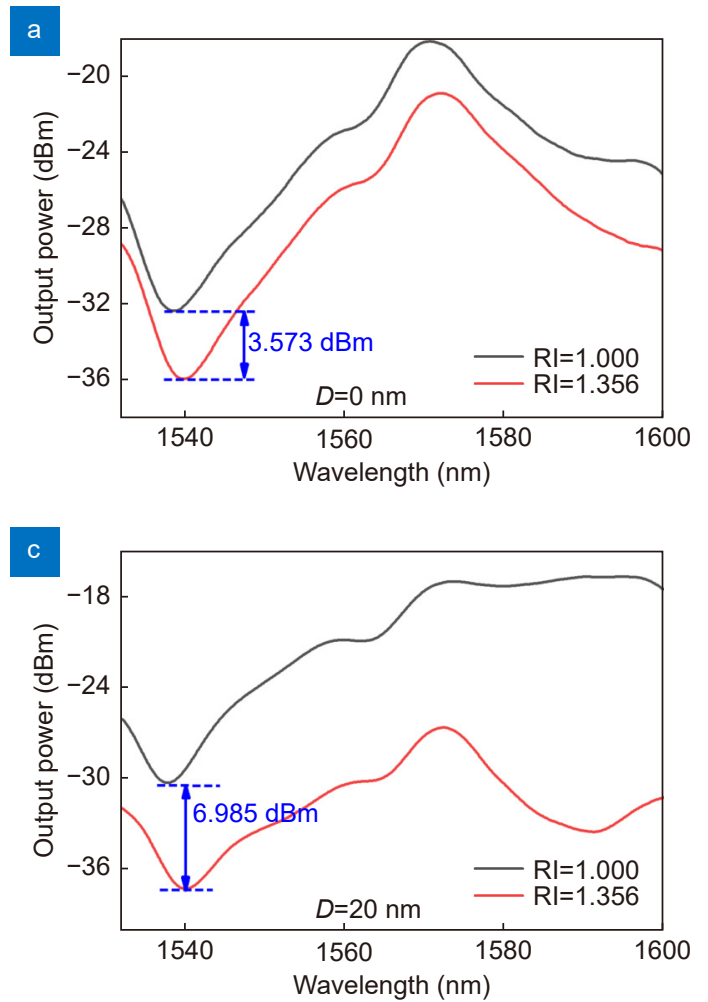

is reduced. As a result, it is not suitable for measurement and observation.

The effect of macro-bending on the sensor is explored. Macro-bending can change the incident angle and the SP-radian. The bending of the sensor with the same direction and SP-radian is defined as the same direction bending (SDB), as shown in Fig. 9(a); the bending of the sensor and with the opposite direction and SP-radian direction is defined as the opposite direction bending (ODB), as shown in Fig. 9(b).

Figure 10 shows the transmission spectra in air and glycerin aqueous solution $(R I=1.356)$ under different bending directions and radius conditions when the PFPOF length is $8 \mathrm{~cm}$ and the SP-depth is $20 \mu \mathrm{m}$. In the environment with same RI difference, the intensity sensitivity increases with the decrease of bending radius. The intensity change of Fig. 10(a) is $7.688 \mathrm{dBm}$, which is higher than $7.012 \mathrm{dBm}$ in Fig. 10(b); the intensity drift of Fig. 10(c) is $12.750 \mathrm{dBm}$, which is higher than $9.068 \mathrm{dBm}$ in Fig. 10(d). The sensitivity of the sensor can be improved by bending with proper radius. Besides, it can also be seen that the performance of SDB is better than that of ODB. With the decrease of bending radius, the mode of light gradually changes from higher order to lower order, and the higher-order modes gradually leak
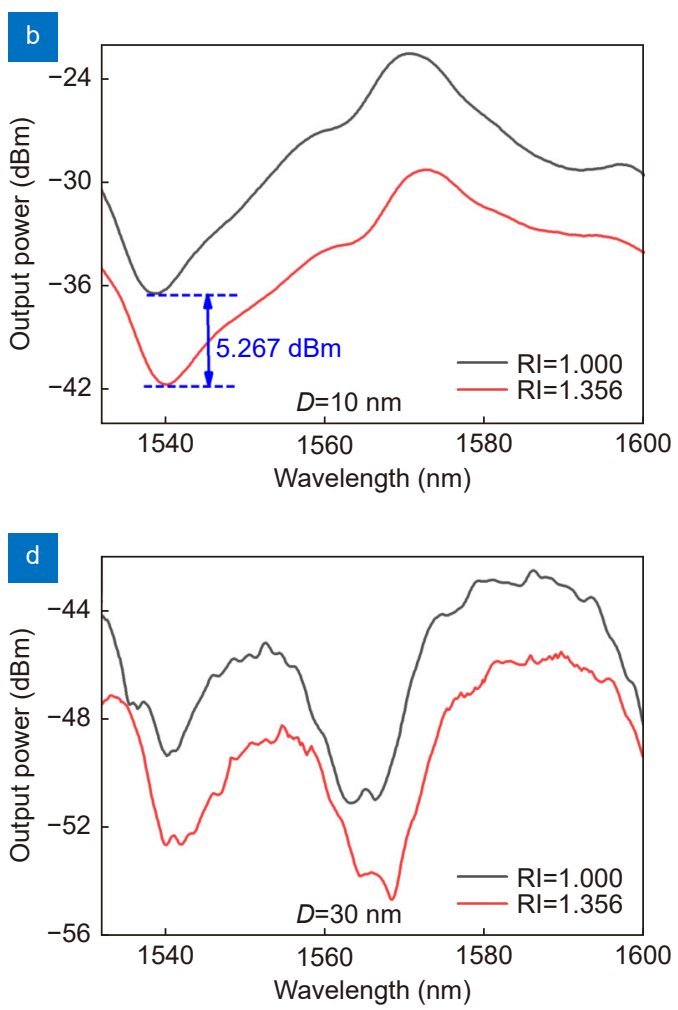

Fig. 8 | Transmission spectra of different SP-depths. (a) $D=0 \mu \mathrm{m}$. (b) $D=10 \mu \mathrm{m}$. (c) $D=20 \mu \mathrm{m}$. (d) $D=30 \mu \mathrm{m}$. 


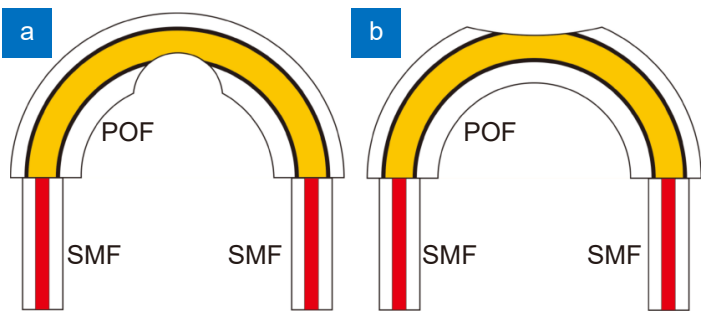

Fig. 9 | Macro-bending diagram. (a) SDB. (b) ODB.

out from the core. As a result, the loss increases, the overall intensity of the output spectrum decreases and the wavelength sensitivity increase ${ }^{25}$. In addition, bending changes the RI distribution of PF-POF, thus changing the mode field distribution of the light, resulting in spectral shifts.

The transmission spectrum intensity of Fig. 10(e) and Fig. 10(f) is weak and the signal-to-noise ratio is reduced, which indicates that the ultra-small bending radius is not good for the sensing. As nearly half of fiber is polished, the bending stress on the SP-area and the normal area is not uniform. This phenomenon becomes obvious gradually with the decrease of bending radius. If the bending radius is too small, the PF-POF will change from the ori-
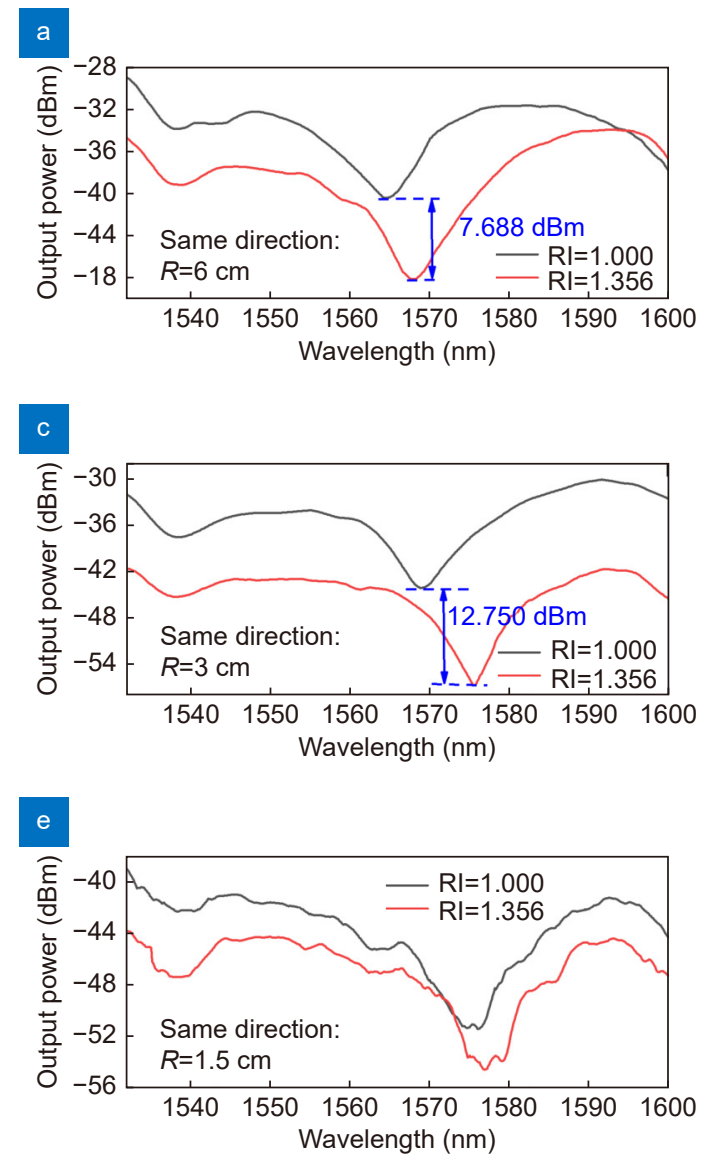

ginal arc shape to the triangular shape, as shown in Fig. 11. This phenomenon leads to the decrease of transmission intensity and signal-to-noise ratio, which is not suitable for measurement and observation. The results show that the SDB structure with $R=3 \mathrm{~cm}$ is a better choice.

A RI sensor with $8 \mathrm{~cm}$ of PF-POF length, $20 \mu \mathrm{m}$ of SPdepth and $3 \mathrm{~cm}$ of SDB radius were fabricated. The RI sensing performance of sensor is studied in the range of $1.330-1.356$ with $0.005 \mathrm{RIU}$ as the interval. As shown in Fig. 12, the black dotted line depicts the nonlinear change trend of the intensity value of the characteristic wavelength in different RI solutions. It can be seen that when the external RI becomes smaller from 1.356 (the relative $\mathrm{RI}$ of the measured solution and the fiber core is 1), the intensity of the characteristic wavelength increases with the decrease of the RI, and the increase rate is becoming larger. This phenomenon verifies the conclusion of the section: Principle. When the relative RI is 1 , all the energy passing through the SP-interface will be refracted out. However, a small amount of light waves in the fiber will not pass through the SP-interface, but is directly coupled to the SMF and detected by the spectrometer, so the intensity of the characteristic wavelength is
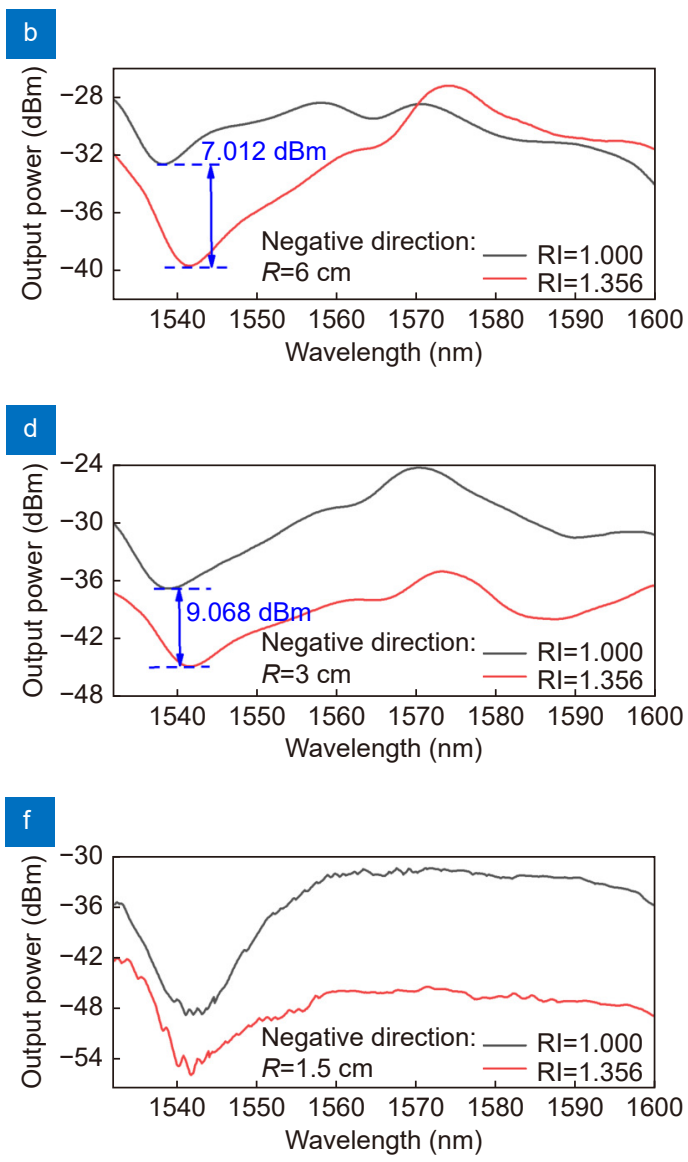

Fig. 10 | Transmission spectra of different macro bending. 


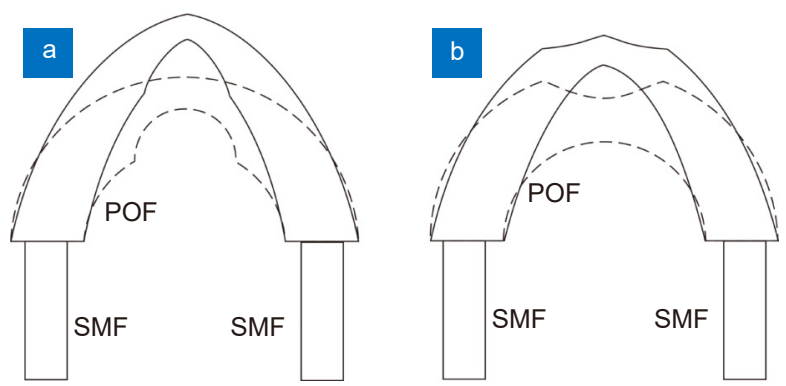

Fig. 11 | Schematic diagram of stress change. (a) SDB. (b) ODB. The dotted line is evenly stressed and the solid line is not evenly stressed.

a

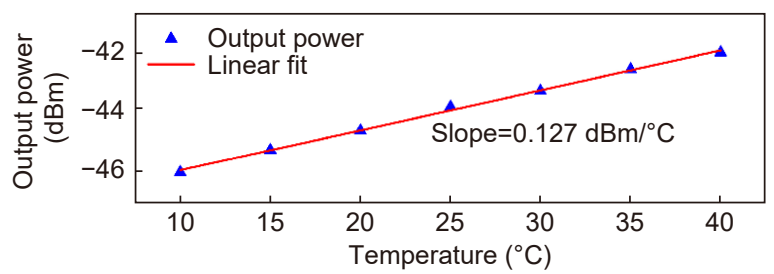

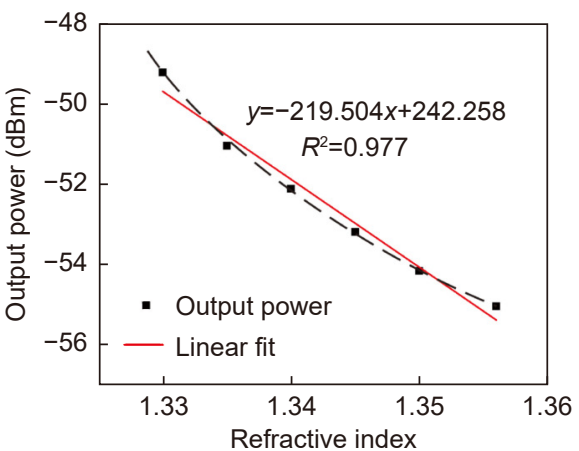

Fig. 12 | Intensity response of RI sensor.

b

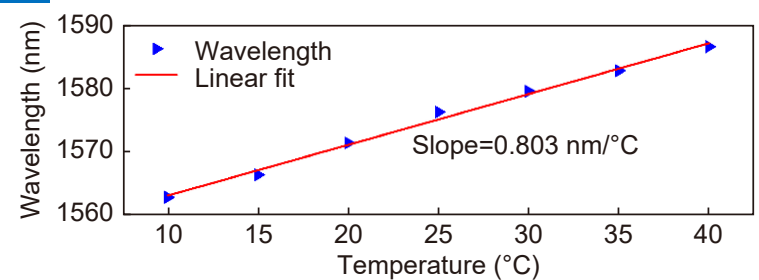

Fig. 13 | Transmission response of the perfluorinated POF-based RI sensor at different temperature. (a) Output power response. (b) Wavelength response.

not zero. The red solid line is the linear fitting of strength response. In the range of $R I=1.330-1.356$, the intensity sensitivity of the sensor is $-219.504 \mathrm{dBm} / \mathrm{RIU}, R^{2}=0.977$.

It can be concluded from theoretical analysis and experimental results that the sensitivity of the sensor has high sensitivity and linearity when the RI of the external solution is less than that of the fiber core. The sensitivity can be improved by properly modifying the structure of the sensor, such as SP and macro-bending.

The temperature characteristics of the RI sensor are researched from $10^{\circ} \mathrm{C}$ to $40^{\circ} \mathrm{C}$. The PF-POF length is 8 $\mathrm{cm}$, the SP-depth is $20 \mu \mathrm{m}$. The sensor is of SDB structure with $R=3 \mathrm{~cm}$. The intensity variation shown in Fig. 13 (a) presents a linear sensitivity of $0.127 \mathrm{dBm} /{ }^{\circ} \mathrm{C}$. The measured wavelength shift presents a linear sensitivity of $0.803 \mathrm{~nm} /{ }^{\circ} \mathrm{C}$ with the temperature rising, as shown in Fig. 13(b). PF-POF has large thermo-optic coefficient and thermal expansion coefficient ${ }^{12}$. The length and numerical aperture of PF-POF will change when temperature changes, which will lead to the change of propagation constant of intrinsic mode and the drift of interference fringes. The results show that the temperature affects the performance of the sensor. In the RI sensing experiment mentioned above, the ambient temperature is stable to reduce the influence of temperature fluctuation and realize high-precision RI measurement. The RI sensor needs temperature compensation before practical application.

\section{Conclusion}

In this work, RI sensor based on PF-POF with different characteristic parameters was successfully fabricated. The sensor with the intensity sensitivity of -219.504 $\mathrm{dBm} / \mathrm{RIU}$ in the range of $R I=1.330-1.356$ was prepared (PF-POF length $=8 \mathrm{~cm}, \mathrm{SDB} \mathrm{R}=3 \mathrm{~cm}$ and SP-depth $=20$ $\mu \mathrm{m})$. The results indicate that the accomplishment of accurate measurement of RI sensor requires appropriate correction and temperature compensation impeded by the large thermo-optic coefficient and the thermal expansion coefficient of PF-POF. Since the perfluorinated plastic optical fiber refractive index sensor has good flexibility, high mechanical strength, simple manufacture and low price, it has wide application prospect in water conservancy, food detection and other fields.

\section{References}

1. Teng HX. Overview of the development of the fluoropolymer industry. Appl Sci 2, 496-512 (2012).

2. Ma H, Jen AKY, Dalton LR. Polymer-based optical waveguides: materials, processing, and devices. Adv Mater 14, 1339-1365 (2002).

3. Bharadwaj R, Sai VVR, Thakare K, Dhawangale A, Kundu T et al. Evanescent wave absorbance based fiber optic biosensor for label-free detection of $E$. coli at $280 \mathrm{~nm}$ wavelength. Biosens Bioelectron 26, 3367-3370 (2011).

4. Wang J, Wang LL. Carbon dioxide gas sensor derived from a 
547-hole microstructured polymer optical fiber preform. Opt Lett 35, 3270-3272 (2010).

5. Han F, Lang TT, Mao BN, Zhao CL, Kang J et al. Surface plasmon resonance sensor based on coreless fiber for high sensitivity. Opt Fiber Technol 50, 172-176 (2019).

6. Chen Q, Liang L, Zheng Q, Zhang Y, Wen L. On - chip readout plasmonic mid - IR gas sensor. Opto-Electronic Adv 3, 190040 (2020).

7. Zhao $Y$, Jin $Y X$, Dong $X Y$, Wang JF. Expermental studies of multimode interference based fiber optic refractive index sensors. Chin J Lasers 37, 1516-1519 (2010).

8. Zhang YJ, Xue LL, Wang TX, Yang L, Zhu B et al. High performance temperature sensing of single mode-multimodesingle mode fiber with thermo-optic polymer as cladding of multimode fiber segment. IEEE Sens J 14, 1143-1147 (2014).

9. Shi QY, Wang YP, Cui YF, Xia W, Guo DM et al. Resolutionenhanced fiber grating refractive index sensor based on an optoelectronic oscillator. IEEE Sens J 18, 9562-9567 (2018).

10. Dong Y, Xiao SY, Wu BL, Xiao H, Jian SS. Refractive index and temperature sensor based on $D$-shaped fiber combined with a fiber Bragg grating. IEEE Sens J 19, 1362-1367 (2019).

11. Chen C, Yu YS, Yang R, Wang C, Guo JC et al. Reflective optical fiber sensors based on tilted fiber Bragg gratings fabricated with femtosecond laser. J Lightwave Technol 31, 455-460 (2013).

12. Zheng $\mathrm{YL}$, Bremer $\mathrm{K}$, Roth $\mathrm{B}$. Investigating the strain, temperature and humidity sensitivity of a multimode graded-index perfluorinated polymer optical fiber with Bragg grating. Sensors 18, 1436 (2018).

13. Zhao Y, Cai L, Hu HF. Fiber-optic refractive index sensor based on multi-tapered SMS fiber structure. IEEE Sens J 15, 6348-6353 (2015).

14. Zhang MZ, Zhu GX, Lu LD, Lou XP, Zhu LQ. Refractive index sensor based on ultrafine tapered single-mode nocladding single-mode fiber structure. Opt Fiber Technol 48, 297-302 (2019).

15. Bhardwaj V, Gangwar RK, Pathak AK, Singh VK. Uncladded sensing fiber for refractive index measurement. In International Conference on Condensed Matter and Applied Physics (AIP Publishing LLC, 2016)
16. Zhou JT, Wang YP, Liao CR, Sun B, He J et al. Intensity modulated refractive index sensor based on optical fiber Michelson interferometer. Sens Actuators B:Chem 208, 315-319 (2015).

17. Zhang $\mathrm{C}, \mathrm{Xu} \mathrm{S}$, Zhao JF, Li HQ, Bai H et al. Intensity-modulated refractive index sensor with anti-light source fluctuation based on no-core fiber filter. Opt Laser Technol 97, 358-363 (2017).

18. Ong YS, Kam W, Harun SW, Zakaria R, Mohammed WS. LowCost Transducer based on surface scattering using side-polished D-shaped optical fibers. IEEE Photon J 7, 7102510 (2015).

19. Liang QT. Physical Optics 4th ed (Electronic Industry Press, Beijing, 2012).

20. Guo YK, Bao PD. Optics Course (Sichuan University Press, China, 1989).

21. Fan XJ, Jiang JF, Zhang XZ, Liu K, Wang S et al. Multimode interferometer-based torsion sensor employing perfluorinated polymer optical fiber. Opt Express 27, 28123-28132 (2019).

22. Socorro AB, Hernaez M, Del Villar I, Corres JM, Arregui FJ et al. Single-mode - multimode - single-mode and lossy mode resonance-based devices: a comparative study for sensing applications. Microsyst Technol 22, 1633-1638 (2016).

23. Feng DJ, Zhang MS, Liu GX, Liu XL, Jia DF. D-shaped plastic optical fiber sensor for testing refractive index. IEEE Sens J 14, 1673-1676 (2014).

24. George NA, Paul AM, Saranya MS. Microbend fiber optic detection of continuously varying refractive index of chlorinated water. Optik 125, 301-303 (2014).

25. Schermer RT. Mode scalability in bent optical fibers. Opt Express 15, 15674-15701 (2007).

\section{Acknowledgements}

We are grateful for financial supports in part by from the National key research and development plan of China (No. 2016YFC0401902), and in part by the National Natural Science Foundation of China (No. U1833104, No. 61775163, No. 61735011, No. 61675152 and No. 61405139).

\section{Competing interests}

The authors declare no competing financial interests. 